\title{
ESPECIE NUEVA DE CERION (MOLLUSCA: PULMONATA: CERIONIDAE) DE HOLGUÍN, CUBA
}

\section{New species of Cerion (Mollusca: Pulmonata: Cerionidae) from Holguín, Cuba}

\begin{abstract}
Alexis Suárez
Sociedad Cubana de Zoología. alexis02@nauta.cu.

\section{RESUMEN}

Se describe la especie nueva Cerion milerae, con localidad tipo en Punta Bejuquera, Gibara, provincia de Holguín, Cuba. Es comparada conquiológicamente con Cerion paucicostatum paucicostatum, Cerion paucicostatum harringtoni y Cerion caroli aedilii. Anatómicamente se compara con Cerion paucicostatum paucicostatum. Con su descripción se incrementa a 148 el número de especies conocidas para Cuba y a 35 el de especies y subespecies en la provincia Holguín. Una extensa prospección en la zona demostró que Cerion milerae está microlocalizada, relacionada al Boniato de costa (Ipomoea pes-caprae), en sustrato arenoso.
\end{abstract}

Palabras clave: Cerion milerae sp. nov., Cerion, Cuba.

\section{ABSTRACT}

Cerion milerae sp. nov. is described from the type locality Punta Bejuquera, Gibara, Holguín province, Cuba. It is compared conchologicaly with Cerion paucicostatum paucicostatum, Cerion paucicostatum harringtoni and Cerion caroli aedilii. Anatomically it is compared with Cerion paucicostatum paucicostatum. With its description the number of species known from Cuba is increased to 148 and to 35 the number of species and subspecies known from Holguín province. An extensive survey in the zone showed that Cerion milerae sp. nov. is microlocalized, associated to Bayhops (Ipomoea pes-caprae), in sandy substrate.

Keywords: Cerion milerae sp. nov., Cerion, Cuba.

\section{INTRODUCCIÓN}

De aproximadamente 600 táxones descritos en el género Cerion Röding, 1798, los que se hallan distribuidos a través de las Antillas Mayores, Trinidad, Curazao, Aruba, Bonaire, Bahamas, Islas Caimanes y el sur de la Florida (Jaume, 1975), 148 pertenecen a la malacofauna cubana. Para la provincia de Holguín, en la región oriental de Cuba, se han descrito 34 morfos, entre especies y subespecies, pertenecientes todas al subgénero Strophiops Dall, 1894 (ver lista).

Todas estas especies poseen rangos de distribución muy restringidos (Fernández et al., 2016); de ellas, C. aguayoi aguayoi, C. blanesi blanesi, C. blanesi bariayi, C. dimidiatum, $C$. josephi josephi, C. microdon microdon, $C$. microdon pygmaeum y $C$. vulneratum vulneratum se localizan en el municipio costero de Gibara. 
Lista de especies y subespecies de Cerion en la provincia Holguín, Cuba

Cerion aguayoi aguayoi Torre y Clench, 1932

Cerion aguayoi bequeaerti Torre y Clench, 1932

Cerion alberti Clench y Aguayo, 1949

Cerion alleni alleni Torre, 1929

Cerion alleni madama Sánchez Roig, 1951

Cerion alleni migueleti Sánchez Roig, 1951

Cerion alleni sanchezi Clench y Aguayo, 1953

Cerion banesense Clench y Aguayo, 1949

Cerion blanesi blanesi Clench y Aguayo, 1951

Cerion blanesi bariayi Aguayo y Jaume, 1957

Cerion crassiusculum crassiusculum Torre, 1899

Cerion crassiusculum smithi Blanes, 1902

Cerion dimidiatum (Pfeiffer, 1847)

Cerion disforme disforme Clench y Aguayo, 1946

Cerion disforme nodalis Clench y Aguayo, 1953

Cerion feltoni Sánchez Roig, 1951

Cerion geophilum Clench y Aguayo, 1949
Cerion hessei Clench y Aguayo, 1949

Cerion humberti Clench y Aguayo, 1949

Cerion josephi josephi Clench y Aguayo, 1949

Cerion microdon microdon Pilsbry y Vanatta, 1896

Cerion microdon pygmaeum Pilsbry y Vanatta, 1896

Cerion nipense Aguayo, 1953

Cerion paucisculptum Clench y Aguayo, 1952

Cerion prestoni Sánchez Roig, 1951

Cerion saetiae Sánchez Roig, 1948

Cerion scalarinum scalarinum Gundlach, 1860

Cerion scalarinum sueyrasi Blanes, 1898

Cerion torrei torrei Blanes, 1898

Cerion torrei moralesi Clench y Aguayo, 1951

Cerion torrei ornatum Pilsbry y Vanatta, 1898

Cerion vulneratum vulneratum (Küster, 1855)

Cerion vulneratum feriai Clench y Aguayo, 1953

Cerion vulneratum lepidium Clench y Aguayo, 1951

\section{OBJETIVO}

-Describir una especie nueva del género Cerion Röding, 1798.

\section{MATERIALES Y MÉTODOS}

Durante trabajo de campo en la zona de Gibara, municipio costero de la provincia de Holguín, se encontró una población de un ceriónido no descrito hasta el momento. Se recolectaron varias conchas que fueron utilizadas para su caracterización, seleccionándose entre ellas la serie tipo.

Entre el material recolectado se incluyeron cuatro ejemplares vivos, utilizados posteriormente para el estudio anatómico en condiciones de laboratorio, cuyos resultados son utilizados en la descripción de la especie. Para este estudio anatómico, los ejemplares seleccionados fueron sumergidos en agua con cristales de menta por un período de tiempo de 24 horas, posteriormente se les practicó una incisión en la región antero-superior, y con una pequeña tijera de disección se separaron los ganglios cerebrales. Se procedió a la extracción del animal de la concha rompiéndose ésta con un fórceps No. 23 de uso estomatológico. Seguidamente, para la obtención de la rádula y su procesamiento, separación del aparato reproductor y toma de medidas, se siguieron los procedimientos utilizados por Suárez (2013). En el caso de la parte blanda del holotipo esta se extrajo luego de sumergirse en el agua con los cristales de menta durante 48 horas, forzado a salir auxiliado por un chorro de agua directamente del grifo. Para el ordenamiento taxonómico de la especie se utilizó la descripción propuesta por Dall (1894).

Abreviaturas. IES: Instituto de Ecología y Sistemática; MNHNCu: Museo Nacional de Historia Natural de Cuba; MHNH: Museo Historia Natural "Carlos de la Torre y Huerta”, de Holguín. 
RESULTADOS

TAXONOMÍA

Clase GASTROPODA, Cuvier, 1795

Subclase PULMONATA Cuvier, 1814

Orden STYLOMMATOPHORA Schmidt, 1856

Familia CERIONIDAE Pilsbry, 1901

Género Cerion Röding, 1798

Subgénero Strophiops Dall, 1894

Cerion milerae sp. nov.

(Figs. 1 y 2)

Material examinado. Se utilizaron las conchas de 14 ejemplares adultos de C. milerae sp. nov., recolectados en diciembre de 2010.

Diagnosis. Concha de color blanco lustroso, ápice ancho, no muy proyectado, de aspecto globoso y costillas que se bifurcan en la última vuelta en las cercanías del ombligo, donde se adentran. No posee diente parietal y el que se halla en la zona columelar es bajo, apenas perceptible. Cerion milerae sp. nov. difiere de Cerion paucicostatum paucicostatum Torre, 1929 y Cerion paucicostatum harringtoni Aguayo y Sánchez Roig, 1953, por poseer una concha más ancha en la porción central, región apical más aguzada y proyectada, costillas más simétricas y algo más unidas, borde peristomal posterior más recto, además de poseer un diente parietal apenas perceptible y la ausencia de diente columelar; en su anatomía, C. paucicostatum paucicostatum difiere de Cerion milerae sp. nov. en su fórmula radular (27-1-27), al igual que en los valores medios de longitud y ancho de su diente central (longitud $=0.019 \mathrm{~mm}$ y ancho $=0.020$ ) y primer lateral (longitud $=0.026 \mathrm{~mm}$ y ancho $=0.022 \mathrm{~mm}$ ), mostrando diferencias además en los valores obtenidos en órganos del aparato reproductor como el divertículo de la bursa copulatrix con $42.4 \mathrm{~mm}$, el atrium con $4.4 \mathrm{~mm}$ de altura y la distancia entre el borde externo del atrium hasta la inserción del epiphalus en el prepucio con $6.1 \mathrm{~mm}$ (Suárez, 2013). De Cerion caroli aedilii Aguayo y de la Torre, 1951, difiere por su forma más corta y obesa, además por mostrar el espacio intercostal más amplio. La mayor diferencia con las restantes especies del grupo radica en la forma globosa de la concha, la distancia del espacio intercostal, y su coloración blanca lustrosa.

Diagnosis (in English). Shell broad and short, with a wide posterior region, shiny white. Ribs fork on the last whorl where they enter the umbilicus. Parietal tooth lacking. Columelar tooth short, hardly perceptible. Cerion milerae sp. nov. it differs from both Cerion paucicostatum paucicostatum Torre, 1929 and Cerion paucicostatum harringtoni Aguayo \& Sánchez Roig, 1953, by having a shell that is broader on it central region, but an apical region that is both, more sharp and projected, ribs that are more symmetrical and joined, last whorl straighter, and a parietal tooth that is scarcely noticeable. C. paucicostatum paucicostatum differs from Cerion milerae sp. nov. in the radular formula (27-1-27), as well as the mean length and width of the central tooth (length $0.019 \mathrm{~mm}$, width 0.020 ), and the first lateral tooth (length $0.026 \mathrm{~mm}$, width $0.022 \mathrm{~mm}$ ). In the reproductive system, the bursa copulatrix diverticulum is $42.4 \mathrm{~mm}$ long, the atrium is $4.4 \mathrm{~mm}$ high, and the distance between the external atrium edge and it insertion on the prepuce is $6.1 \mathrm{~mm}$ (Suárez, 2013). From Cerion caroli aedilii Aguayo \& de la Torre, 1951, it differs because the shorter and wider form, and its wider intercostal space. It is readily distinguished from the rest of the species of Cerion in Cuba by the globular form of the shell, the intercostal distance, and the white glossy color. 


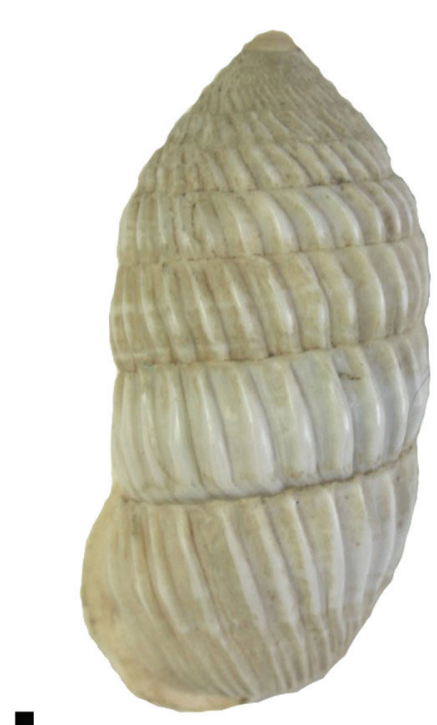

a

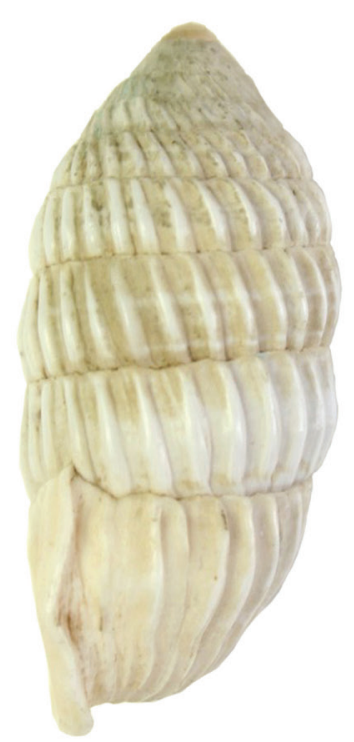

$\mathrm{c}$

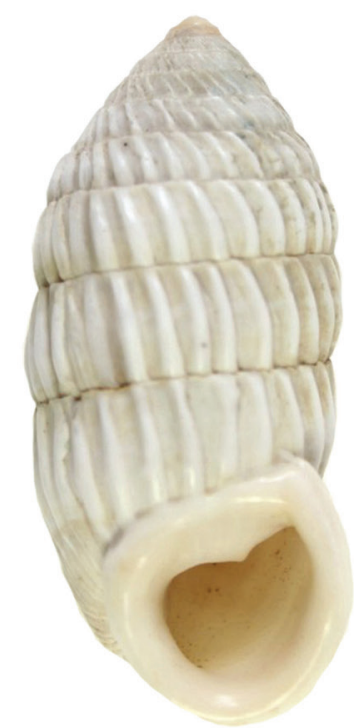

b

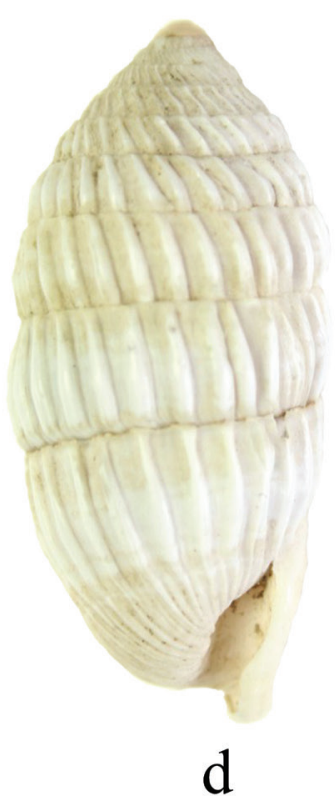




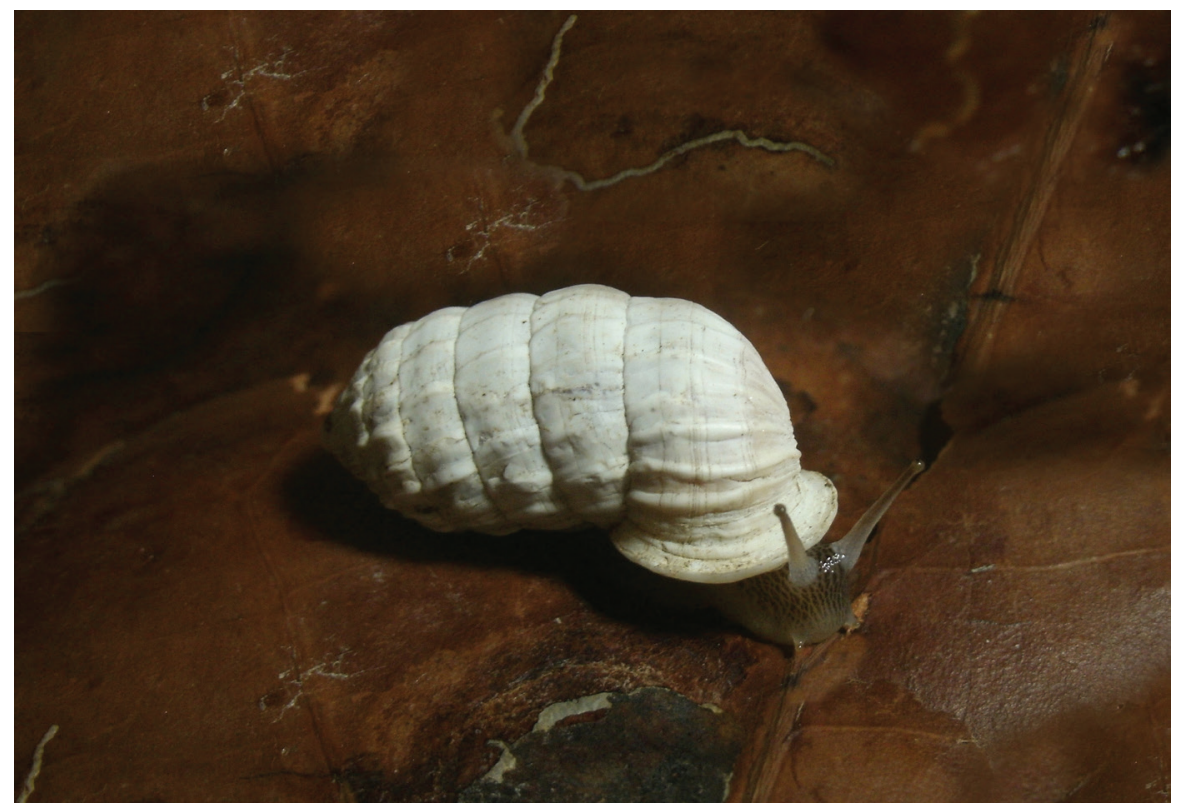

Figura 2. Ejemplar vivo de Cerion milerae sp. nov.

Descripción del Holotipo. Longitud mayor $=33.5 \mathrm{~mm}$; Longitud menor $=31.6 \mathrm{~mm}$; Diámetro mayor $=15.0 \mathrm{~mm}$; Diámetro menor $=15.2 \mathrm{~mm}$; Diámetro mayor de la abertura $=11.0 \mathrm{~mm}$; Diámetro menor de la abertura $=11.7 \mathrm{~mm}$. Concha mediana, sólida, acostillada, de forma subcilíndrica y lados casi paralelos, ligeramente aguzada hacia su extremo anterior y caída casi recta en región posterior, mostrando un color blanco lustroso. En el extremo anterior la protoconcha es comparativamente pequeña de una y media vueltas, de color crema rosa muy pálido, con núcleo marcado que ocupa la mitad de la primera vuelta, a partir de éste la concha es lisa comenzando a marcarse en ella finas y cerradas líneas axiales, dando paso a la teleoconcha sin marcada transición. Teleoconcha de nueve vueltas, algo convexas, separadas por una sutura estrecha y profunda, esculturada con finas líneas axiales no muy altas, estrechas y retroactivas, de bordes romos, separadas por espacios intercostales una y media veces más amplio que el ancho de las costillas, 25 costillas en la última vuelta. Desde la quinta vuelta hacia la zona posterior las costillas axiales comienzan a distanciarse y hacerse más notables apareciendo el color que caracteriza a la concha; en la última vuelta son notables, casi hasta el borde del peristoma, donde son reemplazadas por hilos axiales. Borde interior de la concha de color gris pálido perlado. Abertura elongada, casi semilunar, recta en la porción parietal posterior, donde posee un diente corto y estrecho, casi imperceptible. Peristoma moderadamente engrosado, ligeramente doblado y filoso. Cayo parieto-columelar un poco engrosado y algo expandido. Ombligo profundo, casi circular, hacia donde confluyen todas las costillas de la última vuelta, las que a medida que se introducen en el, se bifurcan y estrechan.

Anatomía del Holotipo. Divertículo de la bursa copulatrix en el sistema reproductor con longitud de $42.375 \mathrm{~mm}$. Atrium de $4.375 \mathrm{~mm}$ de altura, distancia entre el borde externo del atrium hasta la inserción del epiphalus en el prepucio, de $6.087 \mathrm{~mm}$. Fórmula radular 30-1-30 incluyendo las placas marginales $(=61$ dientes por hilera), en la cual los dientes de transición latero-marginales poseen una apariencia variable. Diente central con valores medios de longitud $=0.042 \mathrm{~mm}$ y ancho $=0.045 \mathrm{~mm}$, con una sola cúspide cuyo tamaño es casi la mitad del tamaño del diente. Valores medios del primer diente lateral, longitud $=0.051 \mathrm{~mm}$ y ancho $=0.049 \mathrm{~mm}$, con cúspide estrecha extendida de forma cuadrangular en la base, curvada ligeramente hacia el diente central y una cúspide externa que muestra un borde de base cóncava situado en el límite interior. 
Diente de transición No. 26 con valor medio de longitud $=0.034 \mathrm{~mm}$ y ancho $=0.040 \mathrm{~mm}$, con seis cúspides.

Localidad tipo. Punta Bejuquera, Gibara, Holguín, ( $\left(21^{\circ} 0,8^{\prime} \mathrm{N}\right.$ y $076^{\circ}$ 0,7’ O).

Material tipo. Holotipo, IES: CZACC8. 1. 270 (Fig. 1, a-d); Paratipo, IES: CZACC8. 1. 271. Recolectados en diciembre de 2010, provenientes de la localidad tipo.

Distribución. En esta localidad, la especie tiene un área de distribución microlocalizada.

Etimología. Dedicado a la memoria de José Fernández Milera, naturalista cubano.

Ecología. Los ejemplares de Cerion milerae sp. nov. (Fig. 2), fueron observados en el complejo de vegetación de costa arenosa donde predomina el boniato de costa (Ipomoea pescaprae), en área abierta con exposición total al sol, a unos 200 metros de la línea de mareas.

Comentario. En las colecciones malacológicas depositadas en IES, MNHNCu y MHNH, existen ejemplares conquiológicamente similares que fueron recolectados por el Dr. Carlos de la Torre en Playa Goicuría, Holguín, y etiquetados como Cerion goicurae nomen nodum, pero hasta el momento no han sido descritos. Una prospección reciente realizada en Playa Goicuría con la finalidad de hallar la población anteriormente mencionada, resultó infructuosa.

\section{AGRADECIMIENTOS}

A Iriel Hernández, incomparable amigo e infatigable colega de expediciones, y Alexis Silva, especialista del Museo de Historia Natural de Gibara y excelente guía. A IDEA WILD, por haber donado parte del material utilizado para la obtención de las imágenes. A Robert Cowie, por su ayuda en la traducción de la diagnosis. A José Espinosa, Cary Lixandra, Alejandro Fernández y Esteban Gutiérrez, por la revisión crítica del documento, a Gladis Gil, por su paciencia y ayuda en el trabajo de gabinete. Estos resultados se encuentran incluidos dentro del proyecto Colecciones Zoológicas: su conservación y manejo II, del IES.

\section{LITERATURA CITADA}

Dall, W. H. 1894. Cruise of the Steam Yacht "Wild Duck" in the Bahamas, January to April, 1893, in charge of Alexander Agassiz. II. Notes on the shells collected from the Shores of the Great Lagoon, Watling Island, Bahamas. Bulletin of the Museum of Comparative Zoology, 25 (9): 113-124.

Fernández, A., F. Steffen y A. Suárez. 2016. Restricted range species in the coastal zone of Holguín, Cuba: Checklist and new records of priority species for conservation. Tentacle, 24: 7-10.

Jaume, M. L. 1975. Catálogo de los moluscos terrestres cubanos del género Cerion (Mollusca: Pulmonata: Ceriidae) (con una bibliografía general). Catálogo de la fauna cubana, 37: 1-47.

Suárez, A. 2013. Descripción de Cerion paucicostatum paucicostaum (Mollusca: Pulmonata: Cerionidae). Solenodon, 11: 88-94. 